\title{
Editorial
}

\section{Genomics and breeding of Brassicaceae crops}

Brassicaceae crops include oil crops, vegetables, condiments, fodder crops, and ornamental plants. Most of these crops belong to or are closely related to the genus Brassica. The Brassicaceae also includes the most intensively studied model plant, Arabidopsis thaliana. In molecular genetic studies of Brassicaceae crops, information on A. thaliana genes is highly useful, and many genes important for plant breeding have been identified.

Different species of Brassica have various numbers of chromosomes. The genome relationship of three monogenomic species and three digenomic species that are well known as U's triangle was discovered in Japan, and relationships between different genera have also been studied (Mizushima 1980). In these studies, interspecific and intergeneric hybridizations have been performed, and some interspecific and intergeneric hybrids have been used as breeding materials. Studies on interspecific and intergeneric hybridization are reviewed by Kaneko and Bang in this special issue.

Genome synteny relationships between different species in Brassica and closely related genera are complicated, as exemplified by the different numbers of chromosomes between species. The monogenomic species of Brassica and closely related genera have three copies, on average, of genes homologous to each $A$. thaliana gene, indicating whole genome triplication in the evolution of the ancestral species of Brassica and closely related genera. The recent development of next-generation sequencers has accelerated the determination of whole genome sequences from many species. A draft sequence of the Brassica rapa genome was published in 2011 (Wang et al. 2011), and whole genome sequences of other species of Brassicaceae crops will also be published soon. The progress with genome studies of Brassicaceae crops and genome relationships between them are described by Ashutosh et al.

Many Brassicaceae crops have self-incompatibility, which is not common in other seed-propagated crops. Self-incompatibility is a commonly found trait in many angiosperm species, but self-compatible plants have been selected as crops. The genetics and molecular mechanisms of self-incompatibility have been most intensively studied using Brassica species, and this trait is also used as a tool for producing $\mathrm{F}_{1}$ hybrid seeds of Brassicaceae vegetables. Recent studies on Brassica self-incompatibility are introduced by Kitashiba and Nasrallah. On the other hand, the trait most widely used for $\mathrm{F}_{1}$ hybrid seed production in many crop species is cytoplasmic male sterility. Cytoplas- mic male-sterile lines can be obtained by interspecific hybridization and repeated backcrossing with a paternal species, and this form of sterility is considered to be caused by incompatibility between the cytoplasm and nucleus. Cytoplasmic male sterility in Brassicaceae crops is discussed here by Yamagishi and Bhat.

Brassicaceae crops generally contain glucosinolates, which are degraded to isothiocyanates by the endogenous enzyme myrosinase after disruption of cells. Isothiocyanates are pungent compounds, probably important for defense against herbivores, and they determine the taste or quality of vegetables, condiments, and feed. These compounds are reported to have anti-carcinogenic activity; therefore, Brassica vegetables are in the spotlight for medicinal uses. Studies on glucosinolates in Brassicaceae vegetables are presented by Ishida et al.

Because of water deficit in farmland, probably due to global warming, drought tolerance has become increasingly important in rapeseed breeding. Drought is frequently accompanied by salinity; therefore, recent studies on rapeseed breeding for drought and salt tolerance are reviewed by Zhang et al. Furthermore, herbicide-tolerant cultivars developed by genetic engineering are an increasing percentage of rapeseed produced around the world. For the production of these genetically modified (GM) cultivars, pollen-mediated gene flow from GM cultivars to wild plants or landraces should be limited. Recent studies on the possibility of pollen-mediated gene flow from GM cultivars of rapeseed are described by Tsuda et al.

After the Great East Japan Earthquake, the Rapeseed Project for Restoring Tsunami-Salt-Damaged Farmland was started by Tohoku University because of the high tolerance of Brassica crops to salinity. This project has been supported by Kureha Corporation and other companies. The cost of publication of these reviews is partially supported by this project.

\section{Literature Cited}

Mizushima,U. (1980) Genome analysis in Brassica and allied genera. In: Tsunoda, S., K. Hinata and C. Gomez-Campo (eds.) Brassica crops and wild allies, Japan Sci. Soc. Press, Tokyo, pp. 89-106.

Wang, X., et al. (2011) The genome of the mesopolyploid crop species Brassica rapa. Nature Genet. 43: 1035-1040.

Takeshi Nishio Editor 